Short report

\title{
Physical exercise increases Sestrin 2 protein levels and induces autophagy in the skeletal muscle of old mice
}

\author{
Luciene Lenhare $^{\mathrm{a}, \mathrm{b}, 1}$, Barbara M. Crisol ${ }^{\mathrm{a}, 1}$, Vagner R.R. Silva ${ }^{\mathrm{a}, \mathrm{b}}$, Carlos K. Katashima ${ }^{\mathrm{b}}$, André V. Cordeiro ${ }^{\mathrm{a}}$, \\ Karina D. Pereira c,d , Augusto D. Luchessi ${ }^{\text {c,d }}$, Adelino S.R. da Silva ${ }^{\text {e,f }}$, Dennys E. Cintra ${ }^{g}$, Leandro P. Moura ${ }^{\text {a,h }}$, \\ José R. Pauli a,h, Eduardo R. Ropelle ${ }^{\mathrm{a}, \mathrm{b}, \mathrm{h}, *}$
}

\footnotetext{
a Laboratory of Molecular Biology of Exercise (LaBMEx). School of Applied Sciences, University of Campinas (UNICAMP), Limeira, SP, Brazil

${ }^{b}$ Department of Internal Medicine, Faculty of Medical Sciences, University of Campinas (UNICAMP), Campinas-, SP, Brazil

c Laboratory of Biotechnology, School of Applied Sciences, University of Campinas, 13484-350 Limeira, SP, Brazil

d Institute of Biosciences, São Paulo State University, Rio Claro, SP, Brazil.

e Postgraduate Program in Rehabilitation and Functional Performance, Ribeirão Preto Medical School, USP, Ribeirão Preto, SP, Brazil

${ }^{\mathrm{f}}$ School of Physical Education and Sport of Ribeirão Preto, University of São Paulo, Ribeirão Preto, SP, Brazil

${ }^{g}$ Laboratory of Nutritional Genomics (LabGeN). School of Applied Sciences, University of Campinas, Limeira, SP, Brazil

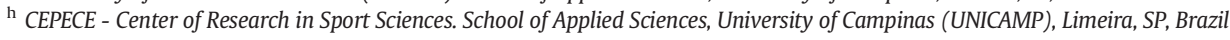

\section{A R T I C L E I N F O}

\section{Article history:}

Received 13 April 2017

Received in revised form 9 July 2017

Accepted 13 July 2017

Available online 17 July 2017

\section{Keywords:}

Physical exercise

Aging

Sestrin 2

Autophagy

Skeletal muscle

\begin{abstract}
A B S T R A C T
Sestrins and autophagy deficiencies are associated with several aging-related organic dysfunctions and metabolic disorders. Here we evaluate the effects of acute exercise on Sestrin 2 (Sesn2) protein content and autophagy markers in the skeletal muscle of experimental models of aging. Twenty-four months-old C57BL/ $6 \mathrm{~J}$ male mice were submitted to a single bout of swimming exercise and the gastrocnemius muscle was evaluated by Western blot. Transcriptomic and phenotypic analysis were also performed by using strains of genetically-diverse BXD mice. The bioinformatics analysis showed a negative correlation between Sesn2 mRNA levels in the skeletal muscle and body weight gain, plasma triglycerides and fasting glucose and positive correlation with several autophagic markers in the muscle of BXD mice. Consistent with these findings, low levels of Sesn2 protein content were

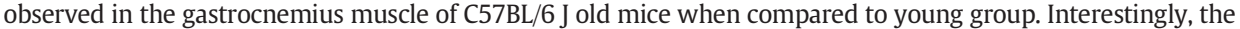
acute aerobic exercise induced Sesn 2 accumulation and modulated several markers of autophagy in the gastrocnemius muscle old mice, including unc-51-like kinase-1 (Ulk1) phosphorylation and the protein levels of Atg5, Atg7, p62 and LC3-II. Finally, exercise increased insulin sensitivity in old animals, as demonstrated by kITT. Taken together, these findings demonstrated the acutely, aerobic physical exercise recovers Sestrin 2 protein content and induces autophagy in the skeletal muscle of old mice, contributing with the improvement of insulin sensitivity an aging animal model.
\end{abstract}

(c) 2017 Elsevier Inc. All rights reserved.

\section{Introduction}

Several stress conditions such as, genomic instability, oxidative stress, hypoxia and fasting, induce the autophagic response in different tissues of mammals. In this scenario, a stress-inducible protein family, Sestrins (SESNs), plays a critical role on autophagy induction under cellular stress conditions (Budanov, 2011; Li et al., n.d.). In addition, it has

\footnotetext{
* Corresponding author at: School of Applied Sciences, University of Campinas (UNICAMP), Limeira, SP, Brazil.

E-mail address: eduardo.ropelle@fca.unicamp.br (E.R. Ropelle).

1 These authors contribute equally to this paper.
}

been demonstrated that Sestrins and autophagy deficiencies are associated with some metabolic disorders in distinct experimental models (Barlow and Thomas, 2015; Kim and Lee, 2014). Lee and colleagues showed that the ablation of drosophila Sestrin (dSesn) resulted in age-related abnormalities, including, triglyceride accumulation, muscle degeneration, mitochondrial and cardiac dysfunction (Lee et al., 2010). Furthermore, autophagic response seems to be affected in the skeletal muscle during aging contributing to sarcopenia (Fan et al., 2016) and insulin resistance (Li et al., n.d.). Thus, pharmacological or physiological strategies to increase or at least maintain the function of Sestrins and the autophagic machinery in the skeletal muscle could be interesting to prevent diverse aging-related diseases (Lee et al., 2013). In the 
present study, we sought to determine the effects of acute aerobic exercise on Sestrin2 protein content and on autophagy in the skeletal muscle of old mice.

\section{Material and methods}

\subsection{Animals and diets}

Male C57BL/6 J mice aged 3 months (young) and 24 months (old) were obtained from the University of Campinas Breeding Center. The investigation was approved by the ethics committee (CEUA 2733-1) and followed the university guidelines for the use of animals in experimental studies and experiments in accordance to the Guide for the Care and Use of Laboratory Animals, published by the U.S. National Institutes of Health (NIH publication no. 85-23 revised 1996). The animals were maintained in $12 \mathrm{~h}: 12 \mathrm{~h}$ artificial light-dark cycles, with lights on at 06:00 a.m., and were housed in 5 animals per cages.

The number of animals used in each experiment is specified in the legend figure.

\subsection{Antibodies}

Antibodies anti-Sesn2 (mouse monoclonal, SC-101249) and Antibodies anti-Atg5 (rabbit polyclonal 32,630), anti-Atg7 (rabbit polyclonal, \#2631), anti-phospho ULK1 (rabbit monoclonal, \#5869), LC3A/B (rabbit, \#4108s), p62 (rabbit, \#5114) and anti-alpha Tubulin (rabbit polyclonal \#2144) from Cell Signalling Technology (Beverly, MA, USA).

\subsection{Exercise protocol}

The animals were habituated in a swimming pool for 10 min during 3 consecutive days. The animals swam in groups of three for two $1 \mathrm{~h}-$ long bouts, separated by a 20 -min rest period, in water maintained at $32{ }^{\circ} \mathrm{C}$. The exercise started at 4:00 p.m. This exercise protocol was adapted from a previously published procedure (Ropelle et al., 2010).

\subsection{Insulin tolerance test}

Eight hours after exercise, mice were submitted to a $30 \mathrm{~min}$ insulin tolerance test. Briefly, $1.5 \mathrm{IU} / \mathrm{kg}$ insulin was injected intraperitoneally and glucose was measured at 0 (basal), 5, 10, 15, 20, 25 and $30 \mathrm{~min}$. The glucose disappearance rate (Kitt) was calculated from the formula $0.693 / t 1 / 2$, where $t 1 / 2$ stands for time for glucose to reach $50 \%$ of the basal value. Glucose $t 1 / 2$ was calculated from the slope of the least square analysis of blood glucose concentration during the linear phase of decline.

\subsection{Tissue sampling}

The mice were anesthetized with intraperitoneal injection of chlorohydrate of ketamine $(50 \mathrm{mg} / \mathrm{kg}$, ketamine, Parke-Davis, Ann Arbor, MI) and xylazine (20 mg/kg, Rompun, Bayer, Leverkusen). After this, the corneal reflexes were verified and assured. After the end of the reflexes, samples of the gastrocnemius, extensor digitorum longus (EDL) and tibialis anterior (TA) were extracted. The tissues were homogenized in extraction buffer (1\% Triton X-100, $100 \mathrm{mM}$ Tris (pH 7.4), $100 \mathrm{mM}$ sodium pyrophosphate, $100 \mathrm{mM}$ sodium fluoride, $10 \mathrm{mM}$ EDTA, $10 \mathrm{mM}$ sodium vanadate, $2 \mathrm{mM}$ PMSF and $0.1 \mathrm{mg}$ of aprotinin $/ \mathrm{mL}$ ) at $4{ }^{\circ} \mathrm{C}$ with a Bead Ruptor 12 Homogenizer (OMNI ${ }^{\circledR}$ ) operated at maximum speed for $60 \mathrm{~s}$. The lysates were centrifuged (Eppendorf® 5804R) at $11000 \mathrm{rpm}$ at $4{ }^{\circ} \mathrm{C}$ for $15 \mathrm{~min}$ to remove insoluble material and the supernatant was used for the assay. The protein content was determined according to the bicinchoninic acid method. Proteins were denatured by boiling in Laemmli sample buffer containing $100 \mathrm{~mol} / \mathrm{l} \mathrm{DTT}$.

\subsection{Western blotting}

The samples were applied in a polyacrylamide gel for separation by SDS-PAGE and transferred to nitrocellulose membranes. The membranes were blocked with $5 \%$ dry milk at room temperature during $1 \mathrm{~h}$ and then incubated with specific antibodies. Specific bands were labeled by chemiluminescence and visualization was performed by exposure of the membranes to RX-films (Kodak ${ }^{\circledR}$ ). The images of protein bands were acquired by the C-DiGit ${ }^{\mathrm{TM} B l o t}$ Scanner (LI-CORR, Lincoln, Nebraska, USA) and quantified using the software UN-SCAN- IT gel. The Western blotting results were normalized by Tubulin and the final values were given in percentage of respective control group. The entire membranes and the statistical analyses of the Western blotting are shown in the supplementary materials (Supplementary. Fig. A.1).

\subsection{Analysis of mRNA content}

Total RNA was isolated using the TRIzol reagent (Invitrogen, Grand Island, NY, USA). A $2 \mu \mathrm{g}$ quantity of total RNA was used as template for the synthesis of cDNA using SuperScript ${ }^{\circledR}$ III First-Strand Synthesis System (Invitrogen). Real-time PCR reactions were performed using $150 \mathrm{ng}$ cDNA, $0.6 \mu \mathrm{M}$ primers (Exxtend, Paulinia, SP, Brazil), and SYBR® Select Master Mix (Applied Biosystem, Warrington, UK). The cycling parameters were: $10 \mathrm{~min}$ at $95^{\circ} \mathrm{C}$ followed by 40 cycles of $15 \mathrm{~s}$ at $95^{\circ} \mathrm{C}, 30 \mathrm{~s}$ at $60{ }^{\circ} \mathrm{C}$, and $30 \mathrm{~s}$ at $72^{\circ} \mathrm{C}$. Relative content of mRNAs was determined after normalization with $\beta 2 \mathrm{M}$ using the ${ }^{\Delta \Delta} \mathrm{Ct}$ method. Each set of primers was designed to recognize unique regions of gene sequences (Supplementary Table A.3).

\subsection{Bioinformatics analysis}

Correlation analyses were performed using a dataset from skeletal muscle of genetically-diverse BXD mice (EPFL/LISP BXD CD Muscle Affy Mouse Gene 1.0 ST (Dec11) RMA ( Log2)) and the phenotype values were obtained using a database, as previously published (Andreux et al., 2012). This database is accessible on Genetwork (http://www. genenetwork.org). The Pearson's correlation graphs were built using PrismGraph and the heat map graph was obtained using the Gene-E software. All individual values used in the bioinformatics analysis are described in the supplementary tables (Supplementary Tables A.1 and A.2).

\subsection{Statistical analysis}

All results were expressed as mean \pm standard error from the mean (SEM). The data was analyzed by Student's $t$-test. The statistical significance used was $p<0.05$. The Pearson's correlations were performed using PrismGraph software and the " $r$ " and " $p$ " values are given in each correlation graph.

\section{Results}

\subsection{Evaluation of Sestrin 2 in the skeletal muscle of BXD and C57BL/6 J old} mice

Initially, we performed a bioinformatics analysis by using a database from several strains of genetically-diverse BXD mice, to evaluate the physiological relevance of Sesn 2 in the skeletal muscle. Interaction analysis remonstrated a negative correlation (red lines) between Sesn2 mRNA in the skeletal muscle and at least three different phenotypes, including, body weight gain $(r=-0.4828, n=42)$, plasma triglycerides $(r=-0.3537, n=42)$ and fasting glucose $(r=-0.280, n=29)$, and a positive correlation (blue lines) between Sesn 2 mRNA and bone mineral mass $(r=0,4084, n=33)$ and body weight loss $(r=0,3361, n=27)$ in BXD mice (Fig. 1A). No correlation (grey lines) was observed between Sesn2 mRNA and rectal temperature $(r=-0.2522, n=22)$, systolic 
A.

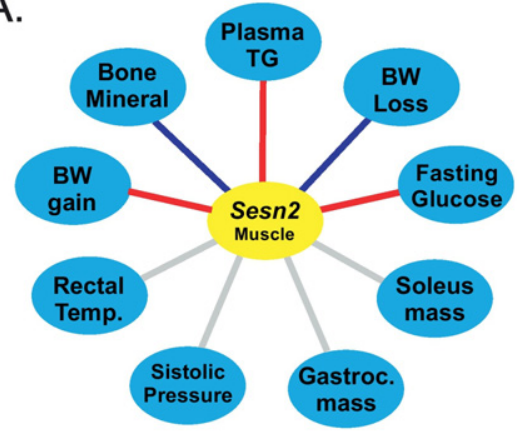

D.

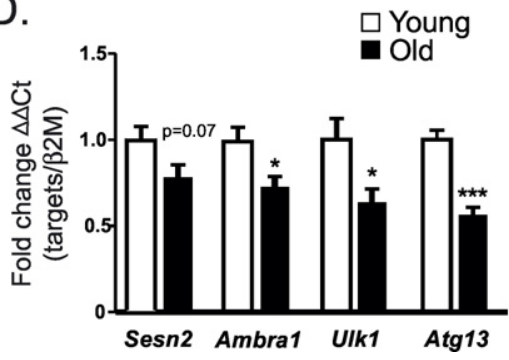

B.

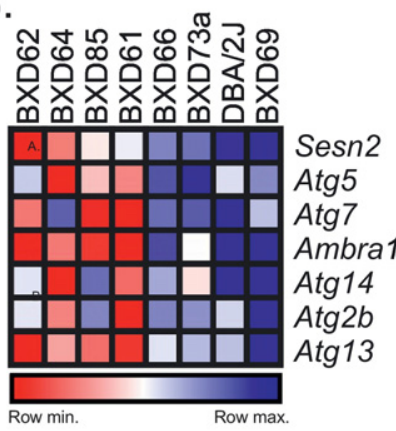

E.

C.

Genetically-diverse BXD mice (muscle)

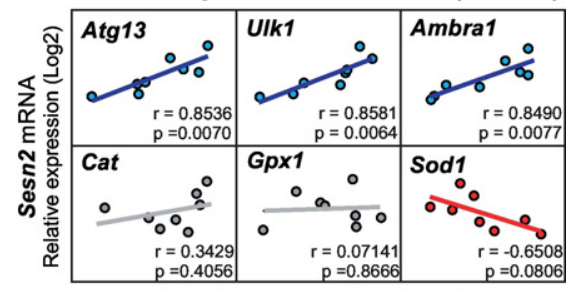

Relative expression (Log2)
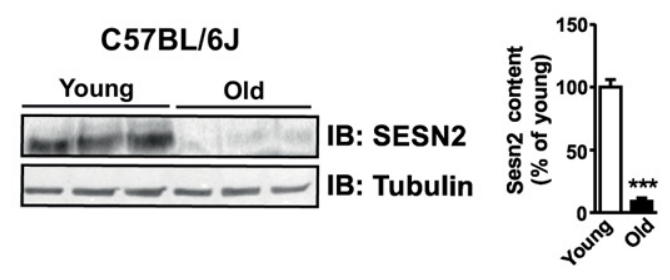

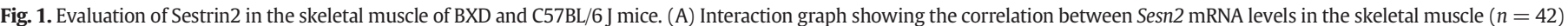

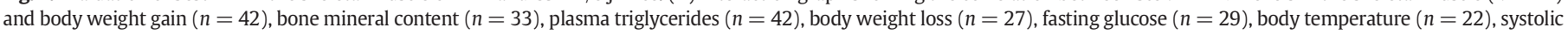

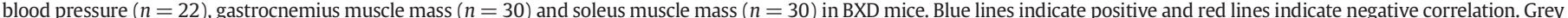

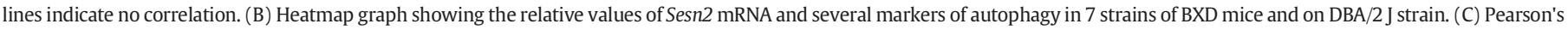

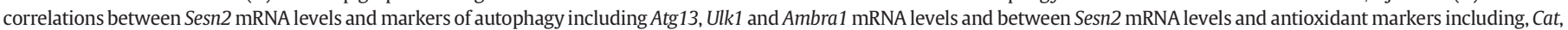

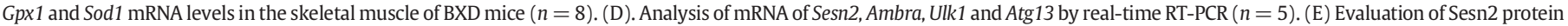
content in the gastrocnemius muscle of young and old mice $(n=3)$. Bars represent the mean \pm S.E.M. ${ }^{*} p<0.05$, vs. young group ${ }^{* * *} p<0.001$, vs. the young group.

blood pressure $(0.1674, n=22)$, gastrocnemius $(r=-0.1153, n=30)$ and soleus muscle mass ( $r=-0.0571, n=30)$ (Fig. 1A).

Specifically in the skeletal muscle, transcriptome analysis revealed that strains containing low levels of Sesn2 mRNA display low levels of autophagy markers, such as, Atg5, Atg7, Ambra1, Atg14, Atg2b and Atg13 mRNA, conversely, strains with high levels of Sesn2 mRNA presented high levels of this gene-set related to autophagy (Fig. 1B). Pearson's correlations confirmed a strong correlation between Sesn2 mRNA and Ambra1, Ulk1 and Atg13 mRNA levels in the skeletal muscle of these BXD strains (Fig. 1C), but negative or no correlation between Sesn 2 mRNA and antioxidant enzymes including, catalase (Cat), glutathione peroxidase (Gpx1) and superoxide dismutase (Sod1) (Fig. 1C).

To confirm these bioinformatics data, we evaluated the mRNA levels of Sesn 2 and some markers of autophagy in the skeletal muscle of young (3-months old) and old (24-months old). Indeed, Ambra1, Ulk1 and Atg13 mRNA levels were reduced in the gastrocnemius muscle of old mice (Fig. 1D). In addition, we found a slight reduction of Sesn2 mRNA levels $(p=0,07)$ (Fig. 1D).

Based on these findings and previous works (Budanov, 2011; Li et al., n.d.), we hypothesized that Sestrin2 protein levels could be affected in the skeletal muscle during aging, contributing with aging-related muscle disorders. For this, we evaluated Sesn 2 protein content in the muscle of aging mouse model, as previously described (Bruusgaard et al., 2006). In fact, Western blot analysis confirmed that aging promoted a significant reduction on Sesn2 protein content in the gastrocnemius muscle of old mice, when compared to young group (Fig. 1E).

\subsection{Exercise induces Sesn2 accumulation in the skeletal muscle of old mice}

Next, we sought to determine the effects of acute swimming exercise on Sesn2 expression in the skeletal muscle of young and old mice. The animals were submitted to a single bout of aerobic exercise protocol and muscle samples were obtained eight hours later, as demonstrated in the experimental design (Fig. 2A). We found that acute exercise induced Sesn 2 accumulation in the gastrocnemius of both, young and old mice (Fig. 2B). Exercise also increased Sesn2 protein levels in other muscle types, including, extensor digitorum longus (EDL) and tibialis anterior (TA) in C57BL/6 J old mice (Fig. 2C). In addition, exercise increased Ulk1 phosphorylation, Atg5, Atg7 and LC3-II and decreased p62 protein levels in the skeletal muscle of old mice (Fig. 2D). Beyond the protein levels, we found that exercise also increased Sesn2, and the markers of autophagy mRNA levels in the gastrocnemius muscle (Fig. 2E). Finally, we evaluated the insulin sensitivity in these mice. No difference was found in the fasting glucose between exercised and rested old animals (Fig. 2E). However, exercise increased insulin sensitivity in old exercised mice (Figs. 2F and G). These data demonstrated that exercise recovers Sesn2 content and elicits autophagic machinery in the muscle of old mice.

\section{Discussion}

Sestrin proteins play a critical role in the control of several cell functions, including the autophagy. In particular, Sesn 1 and Sesn 2 drive different cell types to autophagy (Lee et al., 2010; Li et al., n.d.; Zhang et al., 2013). Sestrin proteins stimulate autophagy through the AMP-activated protein kinase (AMPK)/target of rapamycin complex 1 (TORC1) axis. Whereas, stress-induced Sesn accumulation results in the activation of AMPK and inhibition TORC1 (Budanov, 2011; Lee et al., 2010). Interestingly, it has been demonstrated that Sestrin and autophagy are affected in aged organisms (Colman et al., 2009). For the first time, we demonstrated that Sesn 2 content was markedly reduced in the skeletal muscle of old mice. In line with this, low levels of autophagy markers were found in the muscle of these mice. The mechanism by which the aging process suppresses Sesn2 protein levels is unknown and deserves further investigations.

Paradoxically, oxidative stress is considerate an important stimulator of Sestrin accumulation and autophagy induction in multiple cells and organisms (Budanov, 2011; Lee et al., 2010). Thus, we postulated 
A

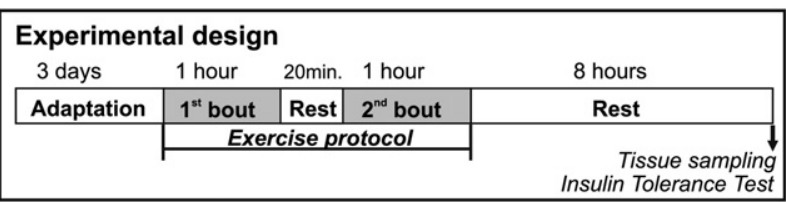

B.

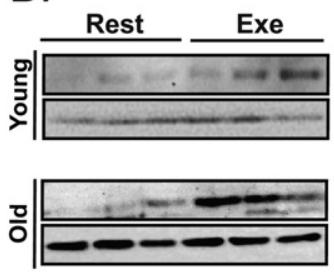

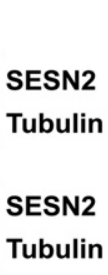

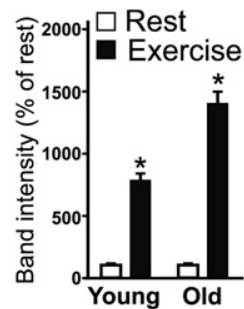

C.
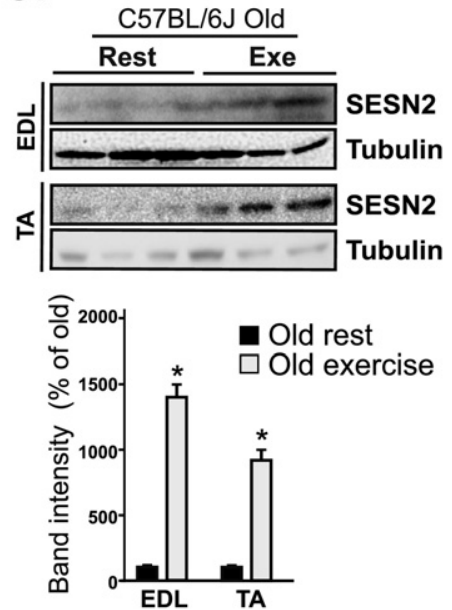

E.

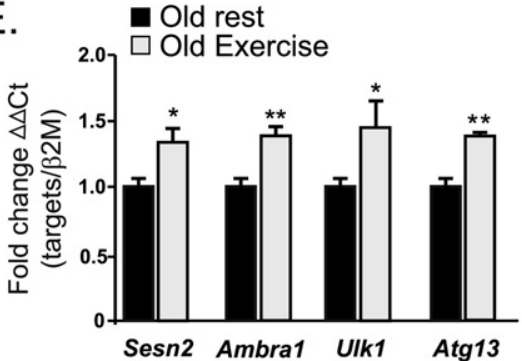

D.

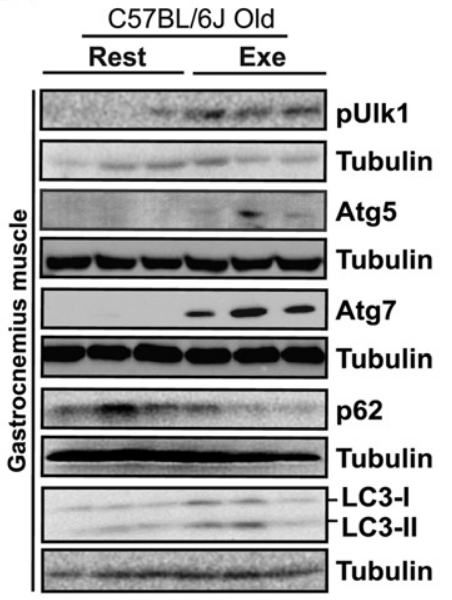

F.

G.

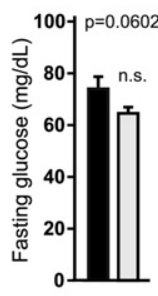

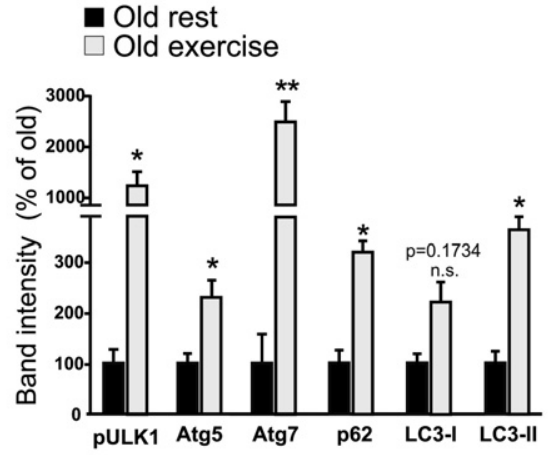

H.

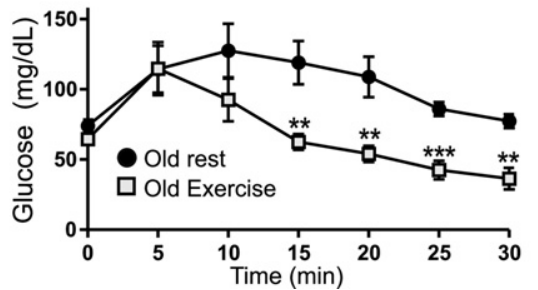

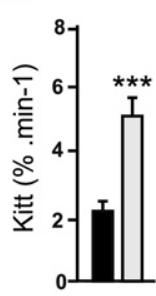

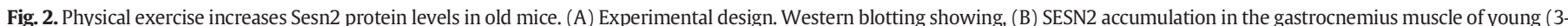

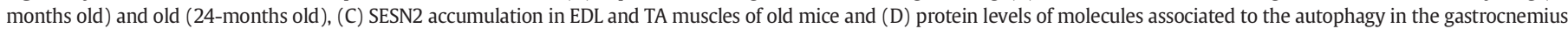

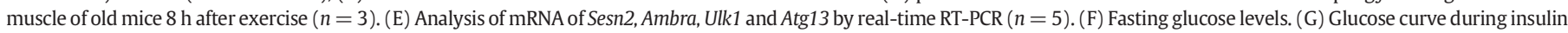
tolerance test. $(\mathrm{H})$ The glucose disappearance rate (Kitt) $(n=6-8)(\mathrm{C})$. Bars represent the mean \pm S.E.M. ${ }^{*} p<0.05,{ }^{* *} p<0.01$, and ${ }^{* * *} p<0.001$ vs. old group at rest.

that exercise could be an interesting alternative to stimulate Sestrin proteins in the skeletal muscle, once; acute exercise induces transitory oxidative stress (Debevec et al., 2017) and autophagy (He et al., 2012; Kim et al., 2013). Few studies have examined the effects of exercise on Sestrin levels. Luciano and colleagues reported that 12 weeks of resistance training increased Sesn 2 protein content in the quadriceps of normal rats (Luciano et al., 2017). Regular exercise increased Sesn 2 and Sesn3 expression in lean and obese mice (Liu et al., 2015). However, the effects of acute physical exercise on the modulation of Sestrins during the aging process are completely unknown. Sesn 2 accumulation in exercised old mice could contribute in different aspects for health, as suggested by our bioinformatics analysis and other studies (Lee et al., 2010; Liu et al., 2015). In the present study, we observed that Sesn2 accumulation and autophagy occurred in parallel to the improvement of insulin sensitivity. Our data are in accordance with previous studies that reported that Sesn2 restores the insulin action in muscle cells (Liu et al., 2015), furthermore, exercise requires the muscle autophagic response to controls the glucose homeostasis in mice (He et al., 2012). In addition, we previously demonstrated that a similar acute swimming exercise protocol increased insulin-induced Insulin Receptor (IR), Insulin Receptor Substrate-1 (IRS-1) and Akt phosphorylation in the skeletal muscle of old mice (Ropelle et al., 2013).
Taken together, the present study demonstrated that acutely, physical exercise stimulates Sesn 2 accumulation and autophagic response in the skeletal muscle of old mice, improving insulin sensitivity in these animals. Overall, these findings provide new insights into the mechanism by which physical activity contributes to the muscle function during the aging.

Supplementary data to this article can be found online at http://dx. doi.org/10.1016/j.exger.2017.07.009.

\section{Author contributions}

L.L. and B.M.C performed all experiments. E.R.R. and L.L. prepared the figures. V.R.R.S.; C.K.K: A.V.C.; supported in protocol exercise, extraction of tissues and Western blot. A.D.L.; and K.D.P. performed the PCR analysis. E.R.R. performed the bioinformatics analysis. L.P.M.; A.S.R.S.; D.E.C. and J.R.P. provided the laboratory support, discussion and reviewed manuscript. L.L. and E.R.R. and wrote the manuscript.

\section{Competing financial interests}

There is no conflict of interest between the authors and the results presented in this study. 


\section{Acknowledgments}

This study was supported by grants from Fundação de Amparo à Pesquisa do Estado de São Paulo (FAPESP- 2016/01089-3) and Coordenação de Aperfeiçoamento de Pessoal de Nível Superior (CAPES).

\section{References}

Andreux, P.A., Williams, E.G., Koutnikova, H., Houtkooper, R.H., Champy, M.-F., Henry, H. Schoonjans, K., Williams, R.W., Auwerx, J., 2012. Systems genetics of metabolism: the use of the BXD murine reference panel for multiscalar integration of traits. Cell 150: 1287-1299. http://dx.doi.org/10.1016/j.cell.2012.08.012.

Barlow, A.D., Thomas, D.C., 2015. Autophagy in diabetes: $\beta$-cell dysfunction, insulin resistance, and complications. DNA Cell Biol. 34:252-260. http://dx.doi.org/10.1089/dna. 2014.2755.

Bruusgaard, J.C., Liestøl, K., Gundersen, K., 2006. Distribution of myonuclei and microtubules in live muscle fibers of young, middle-aged, and old mice. J. Appl. Physiol. 100:2024-2030. http://dx.doi.org/10.1152/japplphysiol.00913.2005.

Budanov, A.V., 2011. Stress-responsive Sestrins link p53 with redox regulation and mammalian target of rapamycin signaling. Antioxid. Redox Signal. 15:1679-1690. http:// dx.doi.org/10.1089/ars.2010.3530.

Colman, R.J., Anderson, R.M., Johnson, S.C., Kastman, E.K., Kosmatka, K.J., Beasley, T.M., Allison, D.B., Cruzen, C., Simmons, H.A., Kemnitz, J.W., Weindruch, R., 2009. Caloric restriction delays disease onset and mortality in rhesus monkeys. Science 325:201-204 http://dx.doi.org/10.1126/science.1173635.

Debevec, T., Millet, G.P., Pialoux, V., 2017. Hypoxia-induced oxidative stress modulation with physical activity. Front. Physiol. 8 (84), 1-9.

Fan, J., Kou, X., Jia, S., Yang, X., Yang, Y., Chen, N., 2016. Autophagy as a potential target for sarcopenia. J. Cell. Physiol. 231:1450-1459. http://dx.doi.org/10.1002/jcp.25260.

He, C., Bassik, M.C., Moresi, V., Sun, K., Wei, Y., Zou, Z., An, Z., Loh, J., Fisher, J., Sun, Q. Korsmeyer, S., Packer, M., May, H.I., Hill, J.A., Virgin, H.W., Gilpin, C., Xiao, G., BasselDuby, R., Scherer, P.E., Levine, B., 2012. Exercise-induced BCL2-regulated autophagy is required for muscle glucose homeostasis. Nature 481, 511-515.
Kim, K.H., Lee, M.-S., 2014. Autophagy as a crosstalk mediator of metabolic organs in regulation of energy metabolism. Rev. Endocr. Metab. Disord. 15:11-20. http://dx.doi. org/10.1007/s11154-013-9272-6.

Kim, Y.A., Kim, Y.S., Oh, S.L., Kim, H.-J., Song, W., 2013. Autophagic response to exercise training in skeletal muscle with age. J. Physiol. Biochem. 69:697-705. http://dx.doi. org/10.1007/s13105-013-0246-7.

Lee, J.H., Budanov, A.V., Park, E.J., Birse, R., Kim, T.E., Perkins, G.A., Ocorr, K., Ellisman, M.H., Bodmer, R., Bier, E., Karin, M., 2010. Sestrin as a feedback inhibitor of TOR that prevents age-related pathologies. Science 327:1223-1228. http://dx.doi.org/10.1126/ science. 1182228 .

Li, H., Liu, S., Yuan, H., Niu, Y., Fu, L., n.d. Sestrin 2 induces autophagy and attenuates insulin resistance by regulating AMPK signaling in C2C12 myotubes. Exp. Cell Res. 354 (1), 18-24. doi:http://dx.doi.org/10.1016/j.yexcr.2017.03.023.

Liu, X., Niu, Y., Yuan, H., Huang, J., Fu, L., 2015. AMPK binds to Sestrins and mediates the effect of exercise to increase insulin-sensitivity through autophagy. Metabolism 64: 658-665. http://dx.doi.org/10.1016/j.metabol.2015.01.015.

Luciano, T.F., Marques, S.D.O., Luiz, B., De, D.R., Araújo, L.V., Nesi, R.T., Scheffer, L., Hugo, V., Pinho, R.A., Muller, A.P., Souza, C.T. De, 2017. Responses of skeletal muscle hypertrophy in Wistar rats to different resistance exercise models. Physiol. Res. 66, 317-323.

Ropelle, E.R., Flores, M.B., Cintra, D.E., Rocha, G.Z., Pauli, J.R., Morari, J., de Souza, C.T., Moraes, J.C., Prada, P.O., Guadagnini, D., Marin, R.M., Oliveira, A.G., Augusto, T.M., Carvalho, H.F., Velloso, L.A., Saad, M.J.A., Carvalheira, J.B.C., 2010. IL-6 and IL-10 antiinflammatory activity links exercise to hypothalamic insulin and leptin sensitivity through IKKß and ER stress inhibition. PLoS Biol. 8, e1000465. http://dx.doi.org/10. 1371/journal.pbio.1000465.

Ropelle, E.R., Pauli, J.R., Cintra, D.E., da Silva, A.S., De Souza, C.T., Guadagnini, D., Carvalho, B.M., Caricilli, A.M., Katashima, C.K., Carvalho-Filho, M.A., Hirabara, S., Curi, R., Velloso, L.A., Saad, M.J.A., Carvalheira, J.B.C., 2013. Targeted disruption of inducible nitric oxide synthase protects against aging, S-nitrosation, and insulin resistance in muscle of male mice. Diabetes 62 (466) (LP-470).

Zhang, X.Y., Wu, X.Q., Deng, R., Sun, T., Feng, G.K., Zhu, X.F., 2013. Upregulation of sestrin 2 expression via JNK pathway activation contributes to autophagy induction in cancer cells. Cell. Signal. 25:150-158. http://dx.doi.org/10.1016/j.cellsig.2012.09.004. 